\title{
Differential regulation of two types of monogalactosyldiacylglycerol synthase in membrane lipid remodeling under phosphate-limited conditions in sesame plants
}

\author{
Mie Shimojima $^{1+}$, Takahide Watanabe ${ }^{2+}$, Yuka Madoka ${ }^{1}$, Ryota Koizumi $^{2}$, Masayuki P. Yamamoto ${ }^{3}$, \\ Kyojiro Masuda ${ }^{4}$, Kyoji Yamada ${ }^{3}$, Shinji Masuda', ${ }^{1}$ and Hiroyuki Ohta, 5, 6 * \\ ' Center for Biological Resources and Informatics, Tokyo Institute of Technology, Yokohama, Japan \\ ${ }^{2}$ Graduate School of Biological Sciences, Tokyo Institute of Technology, Yokohama, Japan \\ ${ }^{3}$ Graduate School of Science and Engineering, University of Toyama, Toyama, Japan \\ ${ }^{4}$ Department of Biology, Faculty of Science, University of Toyama, Toyama, Japan \\ ${ }^{5}$ Earth-Life Science Institute, Tokyo Institute of Technology, Tokyo, Japan \\ ${ }^{6}$ Core Research for Evolutional Science and Technology, Japan Science and Technology Agency, Tokyo, Japan
}

\section{Edited by:}

John Hammond, University of

Reading, UK

\section{Reviewed by:}

Mats X. Andersson, University of Gothenburg, Sweden

Ricarda Jost, The University of

Western Australia, Australia

\section{${ }^{*}$ Correspondence:}

Hiroyuki Ohta, Center for Biological

Resources and Informatics, Tokyo Institute of Technology, 4249-B65

Nagatsuta-cho, Midori-ku, Yokohama, Kanagawa 226-8501, Japan

e-mail:ohta.h.ab@m.titech.ac.jp

${ }^{\dagger}$ Mie Shimojima and Takahide

Watanabe have contributed equally to this work.

\begin{abstract}
Phosphate (Pi) limitation causes drastic lipid remodeling in plant membranes. Glycolipids substitute for the phospholipids that are degraded, thereby supplying Pi needed for essential biological processes. Two major types of remodeling of membrane lipids occur in higher plants: whereas one involves an increase in the concentration of sulfoquinovosyldiacylglycerol in plastids to compensate for a decreased concentration of phosphatidylglycerol, the other involves digalactosyldiacylglycerol (DGDG) synthesis in plastids and the export of DGDG to extraplastidial membranes to compensate for reduced abundances of phospholipids. Lipid remodeling depends on an adequate supply of monogalactosyldiacylglycerol (MGDG), which is a substrate that supports the elevated rate of DGDG synthesis that is induced by low $\mathrm{Pi}$ availability. Regulation of MGDG synthesis has been analyzed most extensively using the model plant Arabidopsis thaliana, although orthologous genes that encode putative MGDG synthases exist in photosynthetic organisms from bacteria to higher plants. We recently hypothesized that two types of MGDG synthase diverged after the appearance of seed plants. This divergence might have both enabled plants to adapt to a wide range of $\mathrm{Pi}$ availability in soils and contributed to the diversity of seed plants. In the work presented here, we found that membrane lipid remodeling also takes place in sesame, which is one of the most common traditional crops grown in Asia. We identified two types of MGDG synthase from sesame (encoded by SeMGD1 and SeMGD2) and analyzed their enzymatic properties. Our results show that both genes correspond to the Arabidopsis type-A and -B isoforms of MGDG synthase. Notably, whereas Pi limitation up-regulates only the gene encoding the type-B isoform of Arabidopsis, low Pi availability up-regulates the expression of both SeMGD1 and SeMGD2. We discuss the significance of the different responses to low Pi availability in sesame and Arabidopsis.
\end{abstract}

Keywords: galactolipid, monogalactosyldiacylglycerol, phosphate deficiency, sesame

\section{INTRODUCTION}

The regulation of galactolipid synthesis in Arabidopsis thaliana under phosphate $(\mathrm{Pi})$-limited conditions has been studied extensively. Under Pi-sufficient conditions, the galactolipids monogalactosyldiacylglycerol (MGDG) and digalactosyldiacylglycerol (DGDG) are found exclusively and abundantly in plastids, especially in thylakoid membranes. They are not observed in other extraplastidial membranes (Joyard etal., 1998). Under Pi-depleted conditions, synthesis of MGDG and DGDG is upregulated, and DGDG is exported from plastids to extraplastidial membranes to help maintain membrane structure after phospholipid degradation (Essigmann et al., 1998; Härtel et al.,
2000; Andersson et al., 2003, 2005; Jouhet et al., 2004). DGDG can substitute for phosphatidylcholine (PC) in the membrane because DGDG and PC are both bilayer-forming lipids, whereas MGDG is not (Murphy, 1986). Regarding the substitution for non-bilayer or anionic phospholipids such as phosphatidylethanolamine and phosphatidylinositol under Pidepleted conditions, glucuronosyldiacylglycerol was suggested to play an essential role (Okazaki et al., 2013).

Although MGDG moiety is unlikely to have a role in PC substitution, MGDG plays an important role as a precursor for DGDG synthesis under Pi-depleted conditions. DGDG is produced by galactosylation of MGDG by the DGDG synthases DGD1 and 
DGD2 in Arabidopsis (Härtel et al., 2000; Kelly and Dörmann, 2002; Kelly et al., 2003). Both DGD1 and DGD2 contribute to DGDG production under Pi-sufficient and Pi-limiting conditions, but the contribution of DGD2 to the increase in extraplastidial DGDG concentrations under Pi-depleted conditions is larger than that of DGD1 (Härtel et al., 2000; Kelly and Dörmann, 2002; Kelly et al., 2003).

There are three MGDG synthases in Arabidopsis that are classified into two types: type A (MGD1) and type B (MGD2 and MGD3; Awai etal., 2001). Type-A MGD1 is mainly involved in the bulk of MGDG synthesis and plays a critical role during the development of chloroplasts and the formation of the photosynthetic apparatus. Analysis of $m g d 1-1$ knockdown and mgd1-2 knockout mutants indicated that the role of MGD1 cannot be fully complemented by MGD2 or MGD3 (Jarvis et al., 2000; Kobayashi et al., 2007, 2013). Under nutrient-sufficient conditions, the expression levels of MGD2 and MGD3 are very low in vegetative tissues (Awai et al., 2001; Kobayashi et al., 2004). The relatively high levels of MGD2 transcripts in non-green tissues such as flowers suggest that galactolipids are likely to have important roles in these organs (Awai et al., 2001; Kobayashi et al., 2004). Indeed, DGDG is a major glycolipid in floral organs, and galactolipid-producing activity is highly up-regulated in the pistils, petals, and elongated pollen tubes of Petunia hybrida and Lilium longiflorum flowers during their development (Nakamura et al., 2003, 2009a). However, the role of type-B MGDG synthase during flower development has not been defined given that neither a $m g d 2$ single nor a $m g d 2 m g d 3$ double knockout mutant displayed any distinctive flower phenotype compared with that of wild-type (WT) plants (Kobayashi et al., 2009a). This suggests that type-A MGDG synthase may complement the function of type-B MGDG synthase in the flower (Kobayashi et al., 2009a). Under nutrient-sufficient conditions, AtMGD3 is relatively highly expressed in roots compared with other organs, but roots of $m g d 3$ plants showed no obvious phenotype compared with WT. These results suggest that type-B MGDG synthases have no crucial role in seedling development under nutrient-sufficient conditions, at least in Arabidopsis.

In contrast, under Pi-depleted conditions, type-B MGDG synthases have an essential role in membrane lipid remodeling (Kobayashi et al., 2009a). MGD2 and MGD3 are main contributors to the increase in DGDG concentrations during Pi starvation by supplying a substrate for DGDG synthesis. Although levels of MGD1 transcripts remain unchanged under Pi-depleted conditions, MGD1 also partially contributes to the increase in DGDG concentrations under Pi-depleted conditions; concentrations of DGDG in leaves are increased in $m g d 2 m g d 3$ under Pi-depleted conditions (Kobayashi et al., 2009a). Moreover, among the two type-B MGDG synthases of Arabidopsis, MGD3 seems to be a main contributor of MGDG synthesis induced by low Pi availability; although developmental defects are observed in mgd3 and $m g d 2 m g d 3$ mutants, no significant defects are observed in the mgd2 mutant (Kobayashi et al., 2009a).

More than a decade has passed since the first isolation of genes encoding type-B MGDG synthases from Arabidopsis, and molecular and biochemical analyses of Arabidopsis mutant plants or WT plants treated with chemical inhibitors of MGDG synthesis have revealed many details of the regulation of MGDG synthesis under Pi-depleted conditions (Botté et al., 2011; Boudière et al., 2012). Besides Arabidopsis, membrane lipid remodeling under Pi-depleted conditions has also been observed in Avena sativa, Glycine max, Phaseolus vulgaris, Acer pseudoplatanus (suspensioncell cultures), Helianthus annuus, Oryza sativa, Raphanus sativus, Tropaeolum majus, Zea mays, and six Proteaceae species (Andersson et al., 2003; Gaude et al., 2004; Jouhet et al., 2004; Russo et al., 2007; Tjellström et al., 2008; Lambers et al., 2012). These data suggest that the up-regulation of galactolipid synthesis following $\mathrm{Pi}$ depletion might be conserved in several higher plants and that it might offer a competitive advantage that allows certain plant species to survive in Pi-limited environments (Kobayashi et al., 2009b; Yuzawa et al., 2012). Indeed, recent comprehensive phylogenetic analyses of genes that encode MGDG synthases in bacteria and plants conducted by our group suggested that a gene encoding a type-B MGDG synthase might have been acquired around the time of the emergence of seed plants; this gene might have been critical to the adaptation of plant species to Pi-limited conditions, which may often have occurred on land during an early phase of the evolution of life on earth (Ohta et al., 2012).

Here we report our findings following analysis of the regulation of MGDG synthesis in sesame, Sesamum indicum L. Sesame is one of the most popular traditional crops in Asia. Sesame plants can grow in soils such as acidic volcanic ash, which suggests their potential suitability as model plants that are relatively tolerant to low available Pi. In this paper, we mainly compared the regulation of MGDG synthesis in sesame with that in Arabidopsis because the regulation of MGDG synthesis at a molecular level is better understood in Arabidopsis than in any of the other higher plants in which this has been analyzed.

\section{RESULTS \\ MORPHOLOGICAL DIFFERENCES BETWEEN SESAME AND ARABIDOPSIS UNDER Pi-DEPLETED CONDITIONS}

Morphology and biochemical processes can be markedly affected when plants are exposed to a Pi-depleted growth environment. Morphological changes, such as growth retardation of shoots and primary roots and elongation of lateral roots and root hairs, have been documented extensively in Arabidopsis plants transferred to Pi-depleted conditions (Bates and Lynch, 1996; Raghothama, 1999; Ticconi and Abel, 2004; Desnos, 2008; Péret et al., 2011; Sato and Miura, 2011). When sesame seedlings were grown under Pidepleted conditions, morphological changes in the lateral roots seemed to be more obvious than those observed in Arabidopsis (Figure 1A). We measured fresh weight per seedling under Pisufficient or -depleted growth conditions for shoots and roots, respectively (Figure 1B). It should be noted that conflicting effects of $\mathrm{Pi}$ depletion have been reported for Arabidopsis. Whereas Kobayashi et al. (2009a) reported that the fresh weight of shoots and that of roots both decreased when plants were grown under Pi-depleted conditions ( $0 \mathrm{mM} \mathrm{Pi}$ in agar medium containing $1 \%$ sucrose), Versaw and Harrison (2002) observed that the fresh weight of shoots decreased but that of roots was unchanged under other Pi-depleted conditions ( $0.2 \mathrm{mM} \mathrm{Pi}$ in agar medium without sucrose). Sucrose in the growth medium enhances the sensitivity of plants to Pi depletion (Lei and Liu, 2011). Given that we used 

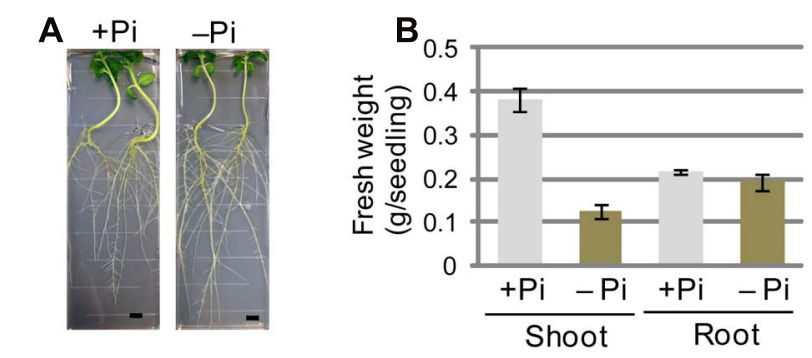

FIGURE 1 | Sesame plants grown under phosphate (Pi)-sufficient and Pi-depleted conditions. (A) Growth phenotypes of sesame seedlings grown on Pi-sufficient medium for 5 days and then on Pi-sufficient (+Pi) or Pi-depleted (-Pi) medium for 7 days. Bars represent $1 \mathrm{~cm}$. (B) Fresh weights of shoots and roots of sesame plants grown on Pi-sufficient medium for 5 days and then on +Pi or -Pi medium for 14 days. Values shown for each of shoots $(n=3)$ and roots $(n=3)$ represent the mean \pm SE for three independent measurements.

the same growth medium as Kobayashi et al. (2009a), we compared the sesame phenotype with their Arabidopsis data.

In sesame, fresh weight of shoots was drastically decreased upon transfer to Pi-depleted medium, similar to the effects shown by Kobayashi et al. (2009a) for Arabidopsis. In contrast, the fresh weight of roots remained unchanged upon transfer to Pi-depleted medium (Figure 1B). These data showed that, when sesame and Arabidopsis are compared, the morphological changes caused by low-Pi stress are similar in shoots but different in roots. The data further suggested that the significant elongation of lateral roots in sesame plants grown under Pi-depleted conditions might offer an advantage for survival under conditions of low Pi availability.

\section{COMPARISON OF THE MEMBRANE LIPID COMPOSITIONS OF SESAME AND ARABIDOPSIS DURING PI STARVATION}

Regarding the lipid compositions of cellular membranes under Pi-depleted conditions, Arabidopsis is known to degrade phospholipids and supply Pi needed for essential biological processes. A simultaneous increase in the concentration of the galactolipid DGDG compensates for the loss of phospholipids from membranes. In both shoots and roots of sesame plants grown under Pi-depleted conditions, the mol\% (relative to total membrane lipids) of DGDG and sulfoquinovosyldiacylglycerol (SQDG) increased and that of phospholipids (phosphatidylglycerol, phosphatidylethanolamine, phosphatidylinositol, and PC) decreased (Figures 2A,B). In shoots, most of the changes were comparable between Arabidopsis and sesame (Figure 2A). However, Pi depletion decreased the phospholipid mol\% (relative to total membrane lipids) by $32 \%$ (from 79 to $47 \%$ ) in sesame roots (Figure 2B). This reduction is slightly larger than the $24 \%$ reduction (from 88 to 64\%) observed in Arabidopsis roots (Kobayashi et al., 2009a). In sesame roots grown under Pi-sufficient conditions, the molar ratio of galactolipids MGDG and DGDG in the total membrane lipids (20\%) was much higher than that in Arabidopsis roots (9\%; Kobayashi et al., 2009a). This difference might result from differences in the abundance of plastids in sesame roots under both of the Pi conditions tested. Exposure of Arabidopsis to Pi-depleted

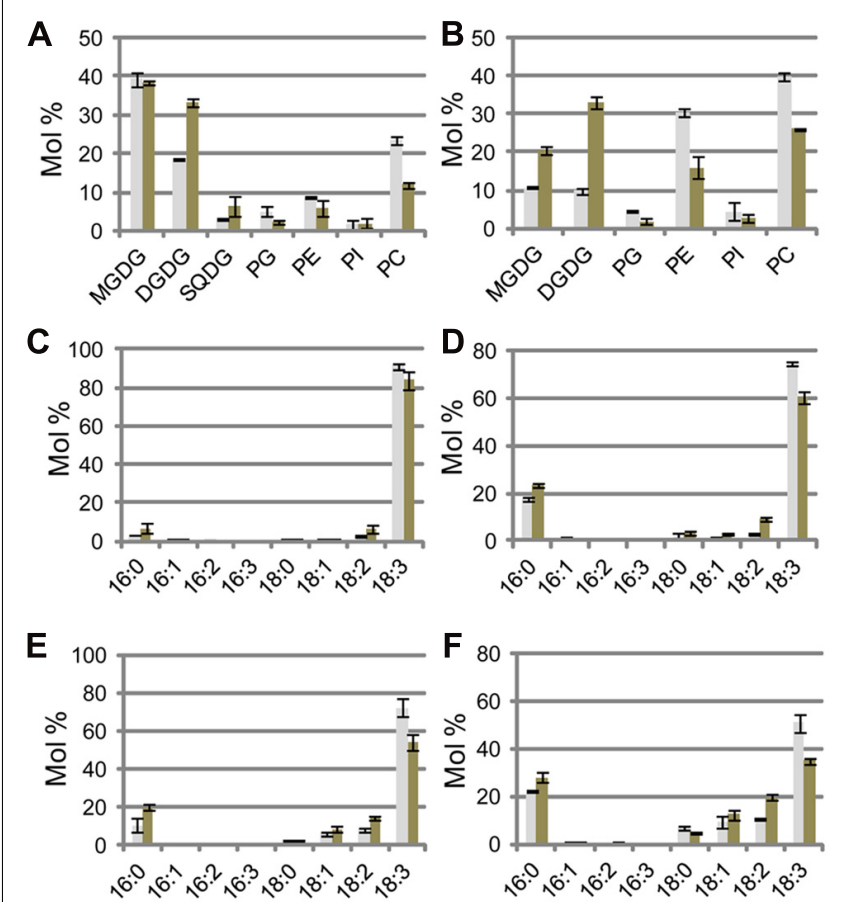

FIGURE 2 | Lipid analysis of sesame seedlings under phosphate (Pi)-sufficient and Pi-depleted conditions. (A,B) Molar ratio of membrane lipids of shoots (A) and roots (B). Fatty acid compositions of MGDG $(\mathbf{C}, \mathbf{E})$ and DGDG (D,F) in shoots (C,D) and roots $(\mathbf{E}, \mathbf{F})$. Gray bars and black bars indicate Pi-sufficient and Pi-depleted conditions, respectively. MGDG, monogalactosyldiacylglycerol; DGDG, digalactosyldiacylglycerol; PC, phosphatidylcholine; PE, phosphatidylethanolamine; PG, phosphatidylglycerol; PI, phosphatidylinositol; SQDG, sulfoquinovosyldiacylglycerol. Values represent the mean \pm SD from three independent measurements.

conditions increases the ratio of DGDG to total acyl lipids in roots but not that of MGDG to total acyl lipids (Kobayashi et al., 2009a). Under Pi-depleted conditions, however, not only were mol\% of DGDG and MGDG in sesame roots increased relative to the respective mol\% under Pi-sufficient conditions, but also the total molar ratio of MGDG and DGDG (combined) reached $53 \%$ of the total membrane lipids, which is $20 \%$ higher than that measured in Arabidopsis roots (Kobayashi et al., 2009a).

Phosphate depletion caused a marked decrease in the abundances of phospholipids in sesame roots. These data suggested that, under Pi-depleted conditions, MGDG synthesis in sesame roots was more strongly up-regulated than in Arabidopsis roots and that the increased supply of Pi from the accelerated degradation of phospholipids might render sesame plants tolerant to low $\mathrm{Pi}$ availability by enabling them to maintain root growth under Pi-depleted conditions.

The effects of Pi depletion on the fatty acid composition of MGDG and DGDG in sesame are shown in Figures 2C-F. Given that MGDG contains no 16:3 fatty acid, which is a signature of "16:3 plants," sesame should clearly be categorized as one of the "18:3 plants," which only possess eukaryotic pathways for lipid synthesis (Figures 2C,D; Heinz and Roughan, 1983; Li-Beisson et al., 2013). Fatty acid compositions of DGDG under both of the Pi conditions tested were similar in sesame (Figures 2E,F). 
Given that DGDG is mainly synthesized from diacylglycerol derived from eukaryotic pathways under both Pi-sufficient and -depleted conditions, the fatty acid composition of DGDG does not differ substantially between Arabidopsis and sesame, although they are distinctly categorized as "16:3 plants" and "18:3 plants," respectively (Figures 2E,F; Kobayashi et al., 2009a).

\section{ISOLATION OF THE TWO GENES THAT ENCODE SESAME MGDG SYNTHASES}

We isolated two genes for sesame MGDG synthase, namely SeMGD1 and SeMGD2 (accession numbers AB841066 and AB841067, respectively). SeMGD1 is a 516-amino acid residue protein that is predicted by ChloroP to contain a chloroplast transit peptide (Emanuelsson et al., 1999) and is categorized as a type-A MGDG synthase on the basis of its similarity to other type-A MGDG synthases (Figure 3). SeMGD2 is a 475-residue protein that is categorized as a type-B MGDG synthase. The failure to identify a putative chloroplast transit peptide in the SeMGD2 sequence suggests that, like AtMGD2 and AtMGD3, SeMGD2 localizes to the outer envelope membrane of chloroplasts. Comparison of the amino acid sequences of SeMGD1 and SeMGD2 with other MGDG synthases (Figure 3) indicated that SeMGD1 and SeMGD2 are highly similar to AtMGD1 (81.3\%) and AtMGD2 (79.6\%), respectively. We could not find the third gene for MGDG synthase by screening a sesame cDNA library. In Arabidopsis, AtMGD2 and AtMGD3 are both involved in MGDG synthesis under Pi-depleted conditions, but AtMGD3 is rather predominantly involved in MGDG synthesis under Pi-depleted conditions (Kobayashi et al., 2009a). Moreover, differences in expression patterns in seedlings suggested that AtMGD2 and AtMGD3 have distinct tissue-specific roles in development, although the $m g d 2$ mutant did not show an apparent phenotype compared with the WT (Awai et al., 2001; Kobayashi etal., 2004). It is possible that sesame might have another type-B MGDG synthase that has another function, given that the cDNA library used was generated from cotyledons of seedlings grown under Pi-sufficient conditions.

\section{REGULATION OF TRANSCRIPT LEVELS OF SeMGDs UNDER Pi-DEPLETED CONDITIONS}

In Arabidopsis, AtMGD1 is constitutively expressed, whereas the transcript abundance of AtMGD2 and AtMGD3 transcripts are markedly increased after transfer to Pi-depleted conditions (Awai et al., 2001; Kobayashi et al., 2004). However, it is not known whether type-B MGDG synthases from other plant species are transcriptionally up-regulated under Pi-depleted conditions. We analyzed the abundance of SeMGD1 and SeMGD2 mRNAs under $\mathrm{Pi}$-sufficient and $\mathrm{Pi}$-depleted growth conditions using quantitative RT-PCR (Figure 4). In sesame, the observed increases in levels of the SeMGD2 transcript under Pi-depleted conditions both in shoots and roots (Figures 4C,D) showed that the transcriptional up-regulation of genes that encode type-B MGDG synthases under Pi-depleted conditions is conserved in plants other than Arabidopsis. Similar to what was observed for AtMGD1, SeMGD1 expression in shoots remained unchanged under Pi-sufficient and Pi-depleted conditions (Figure 4A), but that in roots under Pi-depleted conditions was higher than that under Pi-sufficient conditions (Figure 4B). AtMGD1 is constitutively expressed in various organs, and levels of AtMGD1 transcript are not affected by the Pi concentration in the growth medium (Awai et al., 2001; Kobayashi et al., 2004). Thus, the regulation of MGDG synthesis under Pi-depleted conditions seems to differ between Arabidopsis and sesame.

\section{REGULATION OF SEMGD ACTIVITY BY REDOX STATUS}

Cucumis sativus MGD1 (CsMGD1) was the first MGDG synthase for which the gene was isolated and identified in higher plants (Shimojima et al., 1997). The enzymatic properties of CsMGD1 have been well characterized (Yamaryo et al., 2003, 2006). To verify whether SeMGDs could be regulated by cellular redox status, we measured SeMGD activity under both oxidizing and reducing conditions (Figures 5A,B). The specific activity of SeMGD1 was much higher than that of SeMGD2, and the MGDG synthase activity of each SeMGD1 and SeMGD2 was regulated by redox status. The inactivation of both SeMGD1 and SeMGD2 by oxidization and their activation by reduction suggests that the activities of both type-A and type-B MGD might be also regulated by cellular redox status, as has been shown for CsMGD1 (Yamaryo et al., 2006).

When comparing various sequences of MGDG synthases, we found five highly conserved cysteine residues at positions 277, 291, 383, 418, and 420 of CsMGD1 (Figure 3). Given that our results showed that both types of SeMGDs are regulated by redox state, these five cysteines may be important for enzyme activation.

\section{IN VITRO ACTIVATION OF SESAME TYPE-B MGDG SYNTHASE BY PHOSPHATIDIC ACID}

CsMGD1, spinach MGD1, and AtMGD1 are each activated in vitro by the inclusion of either phosphatidic acid (PA) or phosphatidylglycerol (Covès et al., 1988; Ohta et al., 1995; Dubots et al., 2010). Extensive biological analyses using Arabidopsis concluded that the activation of MGD1 by PA is essential and indispensable for MGDG synthesis in chloroplasts (Dubots et al., 2010). Although the PA-binding residue in the mature protein of AtMGD1 was not determined, the important residues for full activation by PA were previously shown to be P189, H251, W287, and E456 (Dubots et al., 2010; Figure 3). The conservation of all four of these residues in SeMGD1 and SeMGD2, as well as the other MGDG synthases compared in Figure 3, suggests that both SeMGD1 and SeMGD2 might be activated by PA. All published research on the activation by lipids was performed using type-A MGDG synthases. We analyzed activation of type-A and type-B SeMGDs by PA using partially purified recombinant enzymes expressed in Escherichia coli. Intriguingly, both SeMGDs were activated by PA (Figure 6), but again the specific activity of SeMGD1 was much higher than that of SeMGD2. It should be noted that the specific activity of desalted SeMGD1 and SeMGD2 in Figures 5A,B $\left(\sim 6.0 \times 10^{4}\right.$

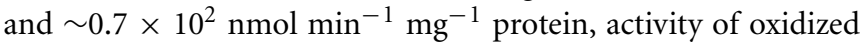
SeMGD1 and SeMGD2 + DTT, respectively) was higher than that of the non-desalted proteins in Figures 6A,B $\left(\sim 0.2 \times 10^{4}\right.$ and $\sim 0.4 \times 10^{2} \mathrm{nmol} \mathrm{min}^{-1} \mathrm{mg}^{-1}$ protein, activity of SeMGD1 and SeMGD2 at $\mathrm{PA}=0 \mathrm{~mol} \%$, respectively). The presence of salts could also explain the difference in PA concentrations required for the maximum activation of both types of SeMGD (10 mol\% for 


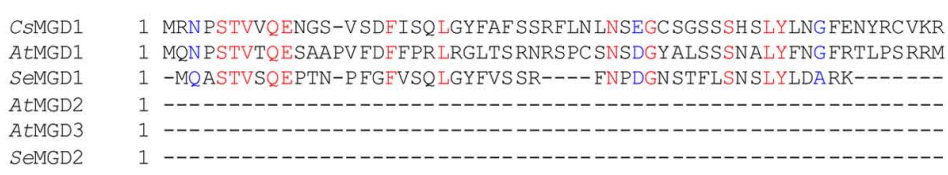

$\begin{array}{lll}\text { CSMGD1 } & 60 \text { PPRSGASLS LSSR-GSSSIRRFVNEFNNVIKFHCHKPPLGFASLG--GVS DETNGI RDD } \\ \text { AtMGD1 } & 61 \text { GKT LASLSFNTKSSAGSSIRRFISDFNSFIRFHCDKVVPESFASVGGVGLS SDENGI REN }\end{array}$ $\begin{array}{lll}\text { AtMGD1 } & 61 & \text { GKT LASLSFNTKSSA GSSLRR FISDFNSFIRFHCDKVVPESFASVGGVGLS SDENGI REN } \\ \text { SeMGD1 } & 48 \text { KPKAVASLS LSTKGAAFSIRRVMNQFNRAIRFHCERIPLGFASVR---VDAGENNGCRDD }\end{array}$

AtMGD2 1 -----------MA TTVMALAEKVLE RVYGTS KSAVSV TSGDGE KTHRHT HHH IHR IKS AtMGD3 1 ---------MMKVVS PRTRSD S ITEKV FRRVYS NFNIST VEDEYI HRQRSS DYEKESRIR SEMGD2 1 ------MFRSSTFI HTTLSECRLDMVMKAASPRKS INRVEERVGVYGFGVVSRQKRCKY

CSMGD1 116 GFGVSODG-ALPLNK IFAENPKRVLLILMSDTGGGHRASAEATKAA FNEEFGNNYOVE ITP AtMGD1 121 GTGGVLGEE GLPLNGVEADRP KKVLILMSDTGGGHRASAEAIRAA FNQEFG DEYQVFITD SEMGD1 105 GNG ILEESDGVPTNGVVSDAPKKVLILMSDTGG GHRASAEAIRAA FNEEFG DDYQVEITD AtMGD2 48 YDDIDEDESSLELIQ IGAERTKNVLILMSDTGGGHRASAEAIRDA FKIEFG DKYRVIVKD AtMGD3 52 KRGLEDKEE VMEMEOMGAFRI KTVLILMSDTGGGHRASAEATRDA FKIEFGDDYRI I I KD SEMGD2 54 AVECEEEDGRMEMEQIGAERT KNVLILMSDTGGGHRASAEATRDAFOLEYGDEYRIFVKD

CSMGD1 175 LWT DHTPWP FNOLPRSYNFLVKHGTTWKMTYYVTAPKVT HQSNFA ATSTFT AREVAKGIN AtMGD1 181 LWT DHTPWP FNOLPR SYNFLVKHGTLWKMTYYGTSPRIVHOSNFAATSTFIAREIAOGIM SEMGD1 165 LWT DHTPWP FNQLPKSYNFLVKHGTLWKMTYYA SAPRVI HRTNFAATSTFI AREVAKGLM AtMGD2 108 VWKEYTGWP LNDMERSYKFMV KHVQLWKVAFHS TSPKWI HSCYLAAIAAYYAKEVEA GLM AtMGD3 112 VWKEYTGWP LNDMERQYKFMV KHVGLWSVAFHGTSPKWI HKSYLSALAAYYAKEIEAGTM SEMGD2 114 VWKEYTGWP LNDMEROYKFMV KHVOLWKVAFHS TSPRWI HSVYLAAFAAFY AKEVEAGIM P189

$$
277 \quad 289291
$$

CSMGD1 235 KYRPDIIISVHPLMQHVPIRI LRSKGLINKIVFTTVVTDLSTCHPTWFHKLVTRCYCPST AtMGD1 241 KYOPDITISVHPLMOHVPT.RVLRSKGLIKKTVFTTVITDTSTCHP TWFHKLVTRCYCPST SEMGD1 225 KYQPDIIIS VHPLMQ HVPIRI LRAKGL IKKIVE TTVVTD ISTCHP TWEHKLVTRCYCPSE AtMGD2 168 EYK PEIIISVHPLMQHIPLWV LKWQEL QKRVLFVTVITD LNTCHP TWFHPGVNRCYC PSQ AtMGD3 172 EYKPDIIISVHPLMQHIPLWVMKWQGL HKKVIFVTVITD INTCHR TWEHHGVSRCYCPSK SEMGD2 174 AYE PDIIISVHPLMQHIPIWV LKRRQNLRKKVTFVTVVTDITTCHR TWEHPS VNRLYY PSE

$\mathrm{H} 251$

${ }^{\Delta}$ W287

CSMGD1 295 EVAKRALTAGLOPSKLKVFGL PVRPSFVKPIRPKIEIRKELGMDENLPAVL LMGGGE GMC AtMGD1 301 EVAKRAQKAGLETSQ I KVYGL PVRPSF VKPVRP KVELRR ELGMDE NLPAVL LMGGGE GMG SeMGD1 285 EVAKRALRA GLQRSQ IKVYGL PVRPSFVKPVRPKDELRKDLGMDE HLPAVL LMGGGEGMG AtMGD2 228 EVAKRALFDGLDESQVRVFGL PVRPSFARAVLVKDDIRKELLEMD DLRAVL LMGGGE GMG AtMGD 3232 EVAKRALVDGLDDSO IRVFGL PVRPSF PRT ILN KNELRKEIEIDL NLPAVL LMGGGE GMG SEMGD2 234 EVAKRALIDGLEESQ IRVFGL PIRPSF CRAVFS KDDLRVELEMDPQLPAVL LMGGGE GMG

CSMGD1 355 PIEATAKAL SKALYYDENHGEP TGQVLVTCGHNKKLAGRLRSTDWKVPVQVKGFVTKMEEC AtMGD1 361 PIEATARALADALYDKNLGEAVGQVLI ICGRNKKLQSKLSSLDWK IPVQVKGEITKMEEC SEMGD1 345 PIEATARALGDALYDETHGEP IGQVLV ICGRNK KLASKLQS INWK I PVEVKGFVSKMEEC AtMGD2 288 PVKETARALEEFLYDKENRKP IGOMVV ICGRNKKLASALEAIDWK IPVKVRGEETOMEKN AtMGD3 292 PVQKTALALGDSLYNSKESNP IGOLIV ICGRNKVLASTLASHEWK IPVKVRGFETOMEKT SEMGD2 294 PVKRTARALGESLFDTKLEKP IGQLI I ICGRNE TLFSTLQSLEWK I PVKIRGFEKQMQKW

$$
418420
$$

CSMGD1 415 MGACDCTITKAGPGT TAFAMT RGLPII LNDYTAGOFAGNVPYVVENGCGKFSKSPKE IAN AtMGD1 421 MGACDCIIT KAGPGT IAEAMI RGLPI I LNGYIA GQEAGNVPYVVE NGCGKF SKSPKE ISK SEMGD 1405 MGACDCIIT KAGPGT IAEAMI RGLPI I LNDYIA GQEAGNVPYVVQ NGCGKF SKSPKE IAN AtMGD2 348 MGACDCIIT KAGPGT IAESLI RSLPI I LNDYIPGQEKGNVPYVVENGAGVETRSPKETAR AtMGD3 352 MGACDCIIT KAGPGT IAEALI CGLPI I LNDYIPGOEKGNVPYVVDNGAGVETRSPKETAK SEMGD2 354 MSACDCIIT KAGPGT I AEAFI RGLPVI LNDY I PGQEKGNVPYVVDNGAGVE TRSPNE TAR
$* *$
E456
$\triangle$

CSMGD1 475 IVAKWFGPKADELLIMSONALRLARPDAVFKIVHDLHELVKORSFVPOYSG-------AtMGD1 481 IVADWFGPA SKEIEIMSONAL RLAKPEAVFKIVHDMHELVRKKNS LPOLSC TA-------

SEMGD1 465 IVS QWFGPKQDELVAMSQNSL RLARPDAVFKIVHDLHELVRQRNFVPQYCT T--------

AtMGD2 408 IVGEWFSTKTDELEQTSDNARKLAQPEEAVFDIVKDIDELSEQRGPLASVSYNLTSSFASI

AtMGD3 412 IVADWFSNNKEEIKKMSENALKLSOPEAVFDIVKDI HHLSOQQORIPLFNE FSY------

\begin{tabular}{|c|c|c|}
\hline CsMGD1 & & - \\
\hline AtMGD1 & & - \\
\hline SeMGD1 & & - \\
\hline AtMGD2 & 468 & \\
\hline MGD3 & & \\
\hline MGD2 & 474 & \\
\hline
\end{tabular}

SEMGD2 414 IVAEWFSTKKDELNRLSENALKLAQPEAVFDIVKDIHDLACQRGPLANISYMLTSSF SSI

FIGURE 3 | Amino acid sequence alignment for MGDG synthases. Red and blue indicate conserved and similar residues, respectively. Identical cysteines are indicated by asterisks; partially conserved cysteines are indicated by open triangles. Four residues in AtMGD1 (P189, H251, W287, and E456) are shown in gray. Cs, Cucumis sativus; At, Arabidopsis thaliana: Se, Sesamum indicum L. Accession numbers are CsMGD (P93115), AtMGD1 (NP_849482), SeMGD1 (AB841066), AtMGD2 (NP_568394), AtMGD3 (NP_565352), and SeMGD2 (AB841067). 


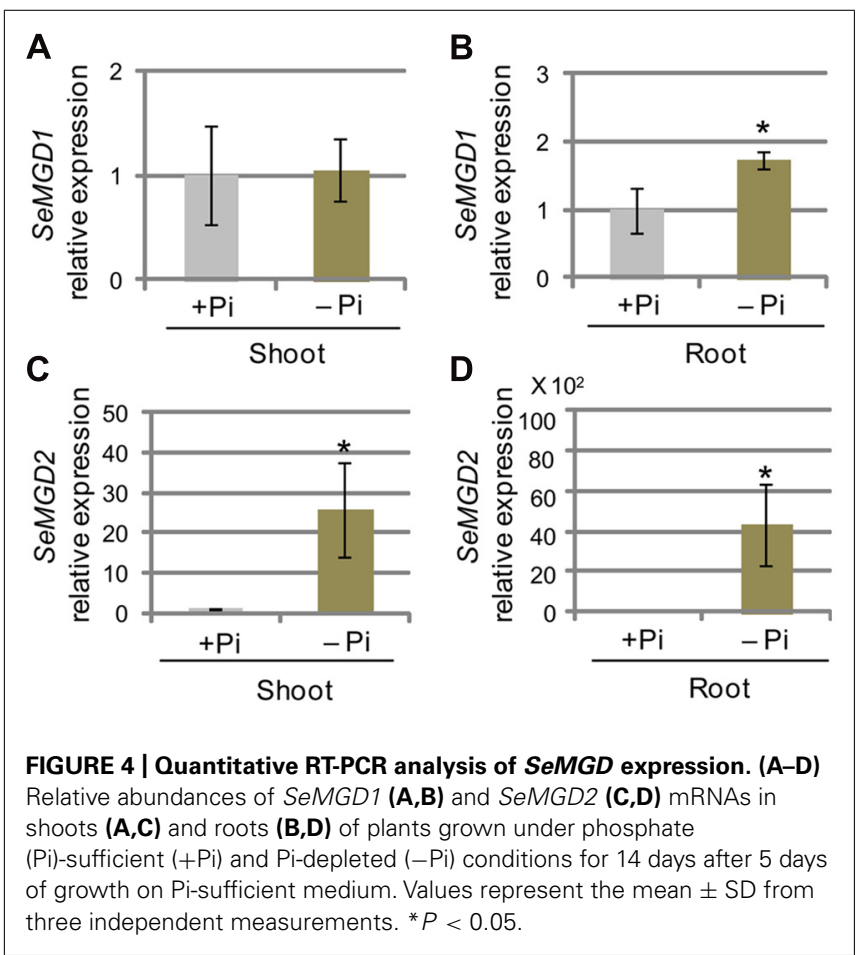

SeMGD1, 18 mol\% for SeMGD2) were substantially higher than those required for AtMGD1 (0.6 mol\%; Dubots et al., 2010).

\section{DISCUSSION}

It is well known that only DGDG, but not MGDG, can substitute for membrane lipids (such as PC) outside of plastids. Given that our data indicate that MGDG accumulates in Pi-depleted roots of sesame plants, we were interested in understanding the distribution of MGDG in roots (Figure 2B). In Arabidopsis, we recently found that severe impairment of thylakoid membrane formation in $m g d 1-2$ is only partially complemented by the synthesis of MGDG and DGDG that is induced by low availability of Pi (Kobayashi etal., 2013). This suggests that only a small part of MGDG and DGDG produced via MGDG synthesis catalyzed by type-B MGDG synthase is able to be transported to thylakoid membranes (Kobayashi et al., 2013). We also found that up-regulation of type-B MGDG synthases under conditions of low Pi availability contributes substantially to DGDG accumulation but only slightly to MGDG accumulation (Kobayashi et al., 2013), probably because type-B MGDG synthase is located in the outer envelope membrane and the MGDG produced could be used immediately for DGDG synthesis by DGD1 and DGD2, which are also located in the outer envelope membrane of plastids. Thus, from these results, we speculate that MGDG accumulated in sesame roots might be derived from the induction of SeMGD1 rather than of SeMGD2 and that MGDG produced in Pi-deficient roots accumulates within plastids.

The level of SeMGD1 expression was slightly increased in roots under $\mathrm{Pi}$-depleted conditions although the magnitude of increase was smaller than that of SeMGD2 in roots and shoots (Figures 4B-D). In Arabidopsis, AtMGD1 mRNA abundance remains unchanged both in shoots and roots under the both $\mathrm{Pi}$
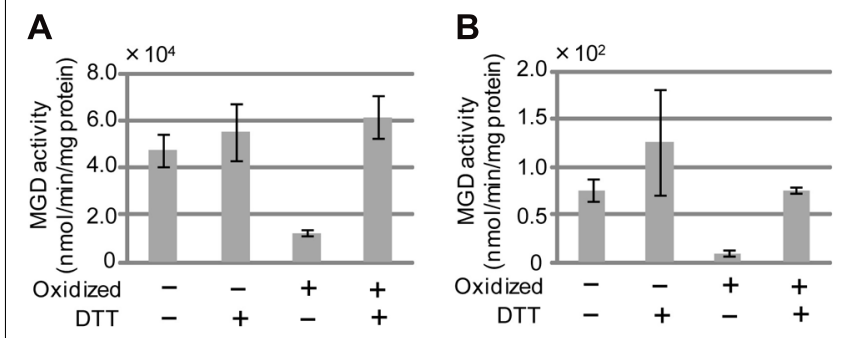

FIGURE 5 | SeMGD activity under reducing and non-reducing conditions. (A) SeMGD1. (B) SeMGD2. Non-oxidized, freshly purified recombinant MGDG synthases from sesame were assayed. Oxidation was performed as described in Materials and Methods. The assay was performed in the presence (+) or absence (-) of dithiothreitol (DTT). Values represent the mean $\pm \mathrm{SD}$ from three independent measurements.

conditions (Awai et al., 2001; Kobayashi et al., 2004). Thus, it is possible that the increase in MGDG concentration in sesame roots was caused by a slight increase in the abundance of the transcript encoding SeMGD1 in roots, i.e., given that the specific activity of SeMGD1 was much higher than that of SeMGD2 (Figures 5 and 6). The distribution of MGDG under Pi-depleted conditions has been controversial. Härtel et al. (2000) reported that MGDG concentrations outside of plastids increase slightly under Pi-depleted conditions even in Arabidopsis. A slight accumulation of MGDG has been observed in the plasma membranes of oat roots under Pi-depleted conditions (Andersson et al., 2003, 2005). It will be interesting to determine where and how MGDG might accumulate in cells under Pi-depleted conditions.

Our data showed that cellular redox status can regulate the activity of both types of SeMGD (Figure 5). It has been suggested that thioredoxin is involved in the regulation of CsMGD1 (Yamaryo et al., 2006). However, we have no data to show that SeMGD activity is regulated by thioredoxin in the same manner as CsMGD1. Given that type-A MGDG synthase is localized in the inner envelope membrane of plastids, we speculate that the activation of type-A MGDG synthase by reduced thioredoxin (photosynthetic activity increases the level of reduction of the chloroplastic pool of thioredoxin) might account for the close correlation between the development of the thylakoid membrane and the maintenance of photosynthetic function. Consistent with this proposal, type-A MGDG synthase is involved in the bulk of MGDG synthesis, and the absence of the corresponding gene severely decreases photosynthetic activity (Jarvis et al., 2000; Kobayashi et al., 2007). On the other hand, the localization of type-B MGDG synthase in the outer envelope membrane of plastids might leave it less affected by the redox status of plastids (Awai et al., 2001; Kobayashi et al., 2009a). Additional research is needed to clarify how regulation of the activity of type-B MGDG synthase by cellular redox status in vivo might be affected by low Pi availability.

Both types of SeMGD were activated by PA in vitro, although the overall specific activity and the required concentration of PA differed markedly between SeMGD1 and SeMGD2 (Figure 6). SeMGD1 was markedly activated by $10 \mathrm{~mol} \% \mathrm{PA}$, whereas SeMGD2 activation required $20 \mathrm{~mol} \% \mathrm{PA}$. We do not know why the activation of MGDG synthases by PA displays such a narrow 

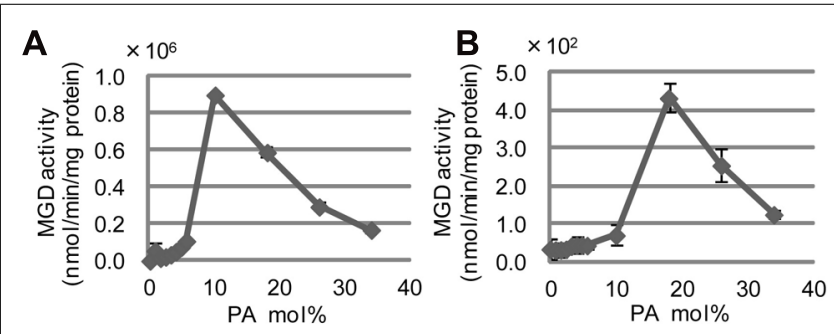

FIGURE 6 | Induction of SeMGD activity by phosphatidic acid (PA).

(A) SeMGD1. (B) SeMGD2. Values represent the mean \pm SD from three independent measurements.

optimum curve, but these concentrations of PA in cell membranes (especially $20 \mathrm{~mol} \% \mathrm{PA}$ ) are not physiologically relevant. Moreover, even after activation by PA, the activity of SeMGD2 was much lower than that of SeMGD1 (Figure 6). Therefore, it seems possible that each SeMGD is activated by PA in vivo through direct interaction with a PA-producing enzyme.

One unsolved problem is the pathway of PA supply for the activation of MGDG synthases. PA could be supplied either from inside or outside of plastids (Li-Beisson et al., 2013). Among the known pathways for PA supply, the only one that might be regulated by $\mathrm{Pi}$ depletion is the pathway that enables PA accumulation through up-regulation of phospholipase $\zeta 2$ (PLD $\zeta 2$ ) abundance during Pi deficiency (Cruz-Ramírez et al., 2006; Acevedo-Hernández et al., 2012). PLD $\zeta 2$ localizes to tonoplasts under Pi-sufficient conditions, but it has been observed that PLD $\zeta 2$-enriched tonoplast domains are preferentially positioned close to mitochondria and adjacent to chloroplasts (Yamaryo et al., 2008). This suggests that the PA produced might activate MGD synthase in the envelope membrane of plastids. Localization of SeMGD2 in the outer envelope membrane, as is the case for Arabidopsis type-B MGDG synthases, might promote the interaction of SeMGD2 with other PA-producing proteins outside of plastids (such as PLD $\zeta 2$ ) because the outer envelope membrane has multiple contact sites with extraplastidial membranes.

The PA pool under Pi-depleted conditions is smaller than that under Pi-sufficient conditions; this is because PA is immediately degraded to diacylglycerol by PA phosphohydrolase 1 and 2 to supply Arabidopsis cells with Pi (Nakamura et al., 2009b). Thus, although it remains uncertain whether SeMGDs are activated in vivo by the cellular pool of PA, it is also possible that these enzymes are activated by other lipids such as phosphatidylglycerol (Dubots et al., 2010). It remains to be resolved whether PA might be a key factor for the activation of type-B MGDG synthase. Several factors besides the availability of Pi might regulate type-B MGDG synthase at the levels of transcript abundance and protein activity in vivo. Kobayashi et al. (2006) evaluated the effect of phosphite on the expression of AtMGD2 and AtMGD3. Phosphite, which is an inactive analog of the $\mathrm{Pi}$ anion, mimics $\mathrm{Pi}$ in signaling pathways. The results showed that increases in the abundances of AtMGD2 and AtMGD3 transcripts was not mediated by the damage to plants induced by a decrease in intracellular Pi. Instead, the increased abundances of AtMGD2 and AtMGD3 transcripts were regulated directly by a Pi-sensing system (Kobayashi et al., 2006).
All of the studies mentioned above have been performed using Arabidopsis. The present study did not involve a similar comparative analysis between sesame and Arabidopsis, but it will be the subject of future work to investigate the key regulator(s) responsible for the activation of type-B MGDG synthase under Pidepleted conditions and to compare the regulatory mechanisms used by higher plants and how they confer tolerance of conditions of limited Pi availability. Using comparative phylogenetic analysis of MGDG synthases, we recently suggested that higher plants developed the capacity for galactolipid synthesis following the acquisition of type-B MGDG synthases on the outer envelope membrane of plastids almost 320 million years ago, which is thought to be immediately after the emergence of Spermatophyta (seed plants; Ohta et al., 2012; Yuzawa et al., 2012). Type-B MGDG synthase mainly supplies MGDG as a precursor for DGDG synthesis under Pi-depleted conditions to facilitate lipid remodeling (Kobayashi et al., 2009a, 2013). This suggests that the acquisition of type-B MGDG synthase may have been one of the key factors that promoted the modern diversity of seed plants on land given that available Pi was likely to become more scarce in some soil types than it was in seawater. Thus, it might be important to use a broad range of plant species to analyze the correlation between the expression level or enzymatic properties of type-B MGDG synthases and the Pi concentration in any given plant species' indigenous habitat. It is also possible that the efficiency of lipid remodeling might have affected species' survival in $\mathrm{Pi}$-limited environments. Thus, comparative analyses of MGDG synthesis among diverse land plants might shed light on the evolution of seed plants.

\section{MATERIALS AND METHODS PLANT MATERIAL AND GROWTH CONDITIONS}

Sesame seedlings ( $S$. indicum L. strain 4294) were grown on Murashige and Skoog medium solidified with $0.8 \%(\mathrm{w} / \mathrm{v})$ agar containing $1 \%(\mathrm{w} / \mathrm{v})$ sucrose for 5 days at $28^{\circ} \mathrm{C}$ under continuous white light, transferred to Pi-sufficient $(1.0 \mathrm{mM})$ or Pi-depleted $(0 \mathrm{mM})$ medium, and grown for another 14 days (Härtel et al., 2000).

\section{LIPID ANALYSIS}

Total lipids were extracted and separated by two-dimensional thin layer chromatography as described by Kobayashi et al. (2007). Lipids isolated from silica gel plates were methylated, and fatty acid methyl esters were quantified by gas chromatography using pentadecanoic acid as an internal standard (Kobayashi et al., 2006).

\section{CONSTRUCTION OF A cDNA LIBRARY}

Total RNA was isolated from sesame cotyledons using an RNA isolation kit (Stratagene). A sesame cDNA library was generated using a cDNA synthesis kit, ZAP-cDNA synthesis kit, and ZAPcDNA Gigapack III gold cloning kit (Stratagene).

\section{ISOLATION OF GENES ENCODING A SESAME MGDG SYNTHASE}

Consensus sequences of type-A MGDG synthase (nucleotide number 540-1378 of AtMGD1 ORF, NM 119327) and typeB MGDG synthase (nucleotide number 527-1184 of AtMGD2 ORF, NM 122048) were selected based on the nucleotide 
sequence alignment of the sequences encoding MGDG synthases from C. sativus (U62622), Nicotiana tabacum (AB047476), O. sativa (AK243359, AB112060, NM 001068022), Spinacia oleracea (AJ249607), and G. $\max (\mathrm{AB} 047475)$. Degenerate primers for nested RT-PCR were constructed; for type-A MGDG synthases: forward $5^{\prime}$-tttntggncngancanacncentggcc- $3^{\prime}$ and reverse $5^{\prime}$-cnttncengententgncengcnatgta- $3^{\prime}$ for the first PCR and forward $5^{\prime}$-ccngatatnatnatcagtgtncatcc- $3^{\prime}$ and reverse $5^{\prime}$ gnccngccttngtnatnatncantcaca- $3^{\prime}$ for the second PCR; for type-B MGDG synthases: forward $5^{\prime}$-gngtncanccnntnatgcaacanattcc- $3^{\prime}$ and reverse $5^{\prime}$-gcnccattntnnacnacatanggnacatt- $3^{\prime}$ for the first PCR and forward $5^{\prime}$-ganctnannacntgncancctacntggtt- $3^{\prime}$ and reverse $5^{\prime}$ tgnnatnatgcantcacangcncccat- $3^{\prime}$ for the second PCR. These PCR fragments were used to screen the sesame cDNA library. The full-length SeMGD1 cDNA was obtained by $5^{\prime}$-RACE using the $5^{\prime}$-Full RACE Core Set (TaKaRa), and that of SeMGD2 cDNA was obtained by $5^{\prime}$-RACE and $3^{\prime}$-RACE using the RNA PCR kit (AMV) Ver. 3.0 (TaKaRa).

\section{QUANTITATIVE RT-PCR}

Total RNA was isolated from three independent sesame samples of shoot and root using the SV Total RNA isolation system (Promega). Reverse transcription was performed using the PrimeScript RT reagent kit (TaKaRa). cDNA was amplified using SYBR PreMix Ex Taq (TaKaRa). Signal detection and quantification were performed in duplicate using the Thermal Cycler Dice Real Time System (TaKaRa). Quantification of SeMGD1 and SeMGD2 transcripts by quantitative PCR was carried out using the SiACT (JP631637) and SiUBQ6 (JP631638) transcript levels for normalization, respectively (Wei et al., 2013). Expression levels were obtained from at least three replicates. The following gene-specific primers were used:

SeMGD1_fw: 5' GGTCTATGGCCTTCCCGTACGG 3'

SeMGD1_rv: 5' TGACTGCAATTTGCTGGCTAGC 3'

SeMGD2_fw: 5' CTAGATGGGCTCGAAGAATCTC 3'

SeMGD2_rv: 5' GATAATCAGTTGTCCGATTGGC 3'

SiACT_fw: 5' GACGAGCTCTGCTGTAGAGAAG 3'

SiACT_rv: 5' CCACCACTGAGAACAATATTGC 3'

SiUBQ6_fw: 5' GATAGGCACTACTGTGGTAAGTG 3'

SiUBQ6_rv: 5' GGAACAGCAATGGTGTCAGCAAC 3'.

\section{PROTEIN EXPRESSION OF SeMGD1 AND SeMGD2 IN E. coli}

SeMGD1 and SeMGD2 were amplified by PCR using a genespecific primer set (forward primer $5^{\prime}$-gccatatggttgatgccggggagaataa$3^{\prime}$ and reverse primer $5^{\prime}$-gcgaattcgtagttgtacaatactgaggtact- $3^{\prime}$ for SeMGD1; forward primer $5^{\prime}$-gccatatgatgttcagaagctctacatttat- $3^{\prime}$ and reverse primer $5^{\prime}$-gcgaattcgtgtaaattaggctcgagaagga- $3^{\prime}$ for SeMGD2) and were then each cloned separately into the pET24a vector. Vectors were transformed into competent cells of E. coli strain BL21(DE3). Transformed cells were grown in Luria-Bertani medium at $37^{\circ} \mathrm{C}$ for $16 \mathrm{~h}$ and diluted 10 -fold using medium to grow for a further $2 \mathrm{~h}$. Protein expression was induced using $1 \mathrm{mM}$ isopropyl thiogalactopyranoside for $3 \mathrm{~h}$ at $37^{\circ} \mathrm{C}$ for $\operatorname{SeMGD1}$ and at $20^{\circ} \mathrm{C}$ for SeMGD2. Cells were collected by centrifugation at $3000 \times g$ for $15 \mathrm{~min}$ and suspended in lysis buffer $(0.1 \mathrm{M}$ MOPS-NaOH, pH 7.8).

\section{PURIFICATION OF SeMGD1 AND SeMGD2 PROTEINS}

Transformed E. coli were resuspended in lysis buffer and disrupted by sonication (Ultrasonic disrupter UD-201, TOMY). Cell lysates were centrifuged at $5000 \times g$ for $3 \mathrm{~min}$ at $4^{\circ} \mathrm{C}$, and the resulting supernatant was centrifuged again at $125,000 \times g$ for $30 \mathrm{~min}$ at $4^{\circ} \mathrm{C}$. Each supernatant was applied to a nickel-charged resin $(\mathrm{Ni}-$ NTA Agarose, Qiagen GmbH), Recombinant MGDG synthase was allowed to bind to the resin at $4^{\circ} \mathrm{C}$ for $30 \mathrm{~min}$, and the resin was washed three times with a three-bed volume of lysis buffer that contained $0.2 \mathrm{M} \mathrm{NaCl}$ and $10 \mathrm{mM}$ imidazole. Each His-tagged MGDG synthase was eluted with lysis buffer containing $0.2 \mathrm{M}$ $\mathrm{NaCl}, 10 \%$ (v/v) glycerol, and $200 \mathrm{mM}$ imidazole to yield a pure protein. In all experiments, protein concentrations were determined using bovine serum albumin as the standard (Bensadoun and Weinstein, 1976).

\section{MEASUREMENT OF MGDG SYNTHASE ACTIVITY}

MGDG synthase activity was measured by determining the amount of $\left[4,5-{ }^{3} \mathrm{H}\right]$ galactose incorporated into the lipid fraction (Yamaryo et al., 2003). Briefly, after pre-incubation of SeMGD in $190 \mu \mathrm{L}$ of assay mixture $(6.4 \mathrm{mM}$ diacylglycerol in $0.01 \%(\mathrm{w} / \mathrm{v})$ Tween 20, $10 \mathrm{mM}$ dithiothreitol, $10 \mathrm{mM}$ sodium acetate, and $0.1 \mathrm{M}$ MOPS-NaOH pH 7.8) at $30^{\circ} \mathrm{C}, 10 \mu \mathrm{L}$ of UDP- $\left[4,5-{ }^{3} \mathrm{H}\right]$ galactose (5.1 mM, $29 \mathrm{~Bq} \mathrm{nmol}^{-1}$ ) was added to initiate the reaction, which was allowed to proceed at $30^{\circ} \mathrm{C}$ for $30 \mathrm{~min}$. The reaction product was quantified using an image analyzer after thin layer chromatography or measured using a liquid scintillation counter.

\section{ACTIVATION OF MGDG SYNTHASE BY PA}

Lipids were removed from the purified SeMGD preparation $\left(1 \mathrm{mg} \mathrm{mL} \mathrm{m}^{-1}\right)$ by stirring at $4^{\circ} \mathrm{C}$ for $30 \mathrm{~min}$ in lysis buffer that contained $24 \mathrm{mM}$ lauryl dimethylamino oxide. MGDG synthase activity was then assessed after the addition of PA using the method described above. PA concentration was calculated as the mol\% of total micelle concentration in the assay mixture (Maréchal et al., 1994).

\section{OXIDATION AND REDUCTION OF MGDG SYNTHASE}

Oxidation and reduction of the SeMGD isoforms were performed according to Yamaryo et al. (2006) with slight modifications. Briefly, the purified MGD was treated with $50 \mu \mathrm{M} \mathrm{CuCl}_{2}$ for $1 \mathrm{~h}$ on ice. The oxidized enzyme was then subjected to gel filtration (ProbeQuant G-50 Micro Column, GE Healthcare) to remove $\mathrm{Cu}^{2+}$ ions and salts and was then used in assays to measure the activity of oxidized MGDG synthase. Reduced MGD was prepared by incubation with $1 \mathrm{mM}$ dithiothreitol at $30^{\circ} \mathrm{C}$ for $30 \mathrm{~min}$.

\section{ACKNOWLEDGMENTS}

This work was supported by a grant-in-aid for Scientific Research on Priority Areas (no. 23119506, no. 25119708, and no. 24580091) and JST CREST from the Ministry of Education, Sports, Science and Culture in Japan.

\section{REFERENCES}

Acevedo-Hernández, G., Oropeza-Aburto, A., and Herrera-Estrella, L. (2012). A specific variant of the PHR1 binding site is highly enriched in the Arabidopsis phosphate-responsive phospholipase DZ2 coexpression network. Plant Signal. Behav. 7, 914-917. doi: 10.4161/psb.20749 
Andersson, M. X., Larsson, K. E., Tjellstrom, H., Liljenberg, C., and Sandelius, A. S. (2005). Phosphate-limited oat. The plasma membrane and the tonoplast as major targets for phospholipid-to-glycolipid replacement and stimulation of phospholipases in the plasma membrane. J. Biol. Chem. 280, 27578-27586. doi: 10.1074/jbc.M503273200

Andersson, M. X., Stridh, M. H., Larsson, K. E., Liljenberg, C., and Sandelius, A. S. (2003). Phosphate-deficient oat replaces a major portion of the plasma membrane phospholipids with the galactolipid digalactosyldiacylglycerol. FEBS Lett. 537, 128-132. doi: 10.1016/S0014-5793(03)00109-1

Awai, K., Maréchal, E., Block, M. A., Brun, D., Masuda, T., Shimada, H., et al. (2001). Two types of MGDG synthase genes, found widely in both 16:3 and 18:3 plants, differentially mediate galactolipid syntheses in photosynthetic and nonphotosynthetic tissues in Arabidopsis thaliana. Proc. Natl. Acad. Sci. U.S.A. 98, 10960-10965. doi: 10.1073/pnas.181331498

Bates, T. R., and Lynch, J. P. (1996). Stimulation of root hair elongation in Arabidopsis thaliana by low phosphorus availability. Plant Cell Environ. 19, 529-538. doi: 10.1111/j.1365-3040.1996.tb00386.x

Bensadoun, A., and Weinstein, D. (1976). Assay of proteins in the presence of interfering materials. Anal. Biochem. 70, 241-250. doi: 10.1016/S0003 2697(76)80064-4

Botté, C. Y., Deligny, M., Roccia, A., Bonneau, A. L., Saïdani, N., Hardré, H., etal. (2011). Chemical inhibitors of monogalactosyldiacylglycerol synthases in Arabidopsis thaliana. Nat. Chem. Biol. 7, 834-842. doi: 10.1038/ nchembio. 658

Boudière, L., Botté, C. Y., Saidani, N., Lajoie, M., Marion, J., Bréhélin, L., et al. (2012). Galvestine-1, a novel chemical probe for the study of the glycerolipid homeostasis system in plant cells. Mol. Biosyst. 8, 2023-2035. doi: 10.1039/c2mb25067e

Covès, J., Joyard, J., and Douce, R. (1988). Lipid requirement and kinetic studies of solubilized UDP-galactose:diacylglycerol galactosyltransferase activity from spinach chloroplast envelope membranes. Proc. Natl. Acad. Sci. U.S.A. 85 4966-4970. doi: 10.1073/pnas.85.14.4966

Cruz-Ramírez, A., Oropeza-Aburto, A., Razo-Hernández, F., Ramirez-Chávez, E., and Herrera-Estrella, L. (2006). Phospholipase DZ2 plays an important role in extraplastidic galactolipid biosynthesis and phosphate recycling in Arabidopsis roots. Proc. Natl. Acad. Sci. U.S.A. 103, 6765-6770. doi: 10.1073/pnas.0600863103

Desnos, T. (2008). Root branching responses to phosphate and nitrate. Curr. Opin. Plant Biol. 11, 82-87. doi: 10.1016/j.pbi.2007.10.003

Dubots, E., Audry, M., Yamaryo, Y., Bastien, O., Ohta, H., Breton, C., et al. (2010) Activation of the chloroplast monogalactosyldiacylglycerol synthase MGD1 by phosphatidic acid and phosphatidylglycerol. J. Biol. Chem. 285, 6003-6011. doi: 10.1074/jbc.M109.071928

Emanuelsson, O., Nielsen, H., and Von Heijne, G. (1999). ChloroP, a neural networkbased method for predicting chloroplast transit peptides and their cleavage sites. Protein Sci. 8, 978-984. doi: 10.1110/ps.8.5.978

Essigmann, B., Güler, S., Narang, R. A., Linke, D., and Benning, C. (1998). Phosphate availability affects the thylakoid lipid composition and the expression of SQD1, a gene required for sulfolipid biosynthesis in Arabidopsis thaliana. Proc. Natl. Acad. Sci. U.S.A. 95, 1950-1955. doi: 10.1073/pnas.95.4.1950

Gaude, N., Tippmann, H., Flemetakis, E., Katinakis, P., Udvardi, M., and Dörmann, P. (2004). The galactolipid digalactosyldiacylglycerol accumulates in the peribacteroid membrane of nitrogen-fixing nodules of soybean and lotus. J. Biol. Chem. 279, 34624-34630. doi: 10.1074/jbc.M404098200

Härtel, H., Dörmann, P., and Benning, C. (2000). DGD1-independent biosynthesis of extraplastidic galactolipids after phosphate deprivation in Arabidopsis. Proc. Natl. Acad. Sci. U.S.A. 97, 10649-10654. doi: 10.1073/pnas.180320497

Heinz, E., and Roughan, P. G. (1983). Similarities and differences in lipid metabolism of chloroplasts isolated from 18:3 and 16:3 plants. Plant Physiol. 72, 273-279. doi: 10.1104/pp.72.2.273

Jarvis, P., Dörmann, P., Peto, C. A., Lutes, J., Benning, C., and Chory, J. (2000) Galactolipid deficiency and abnormal chloroplast development in the Arabidopsis MGD synthase 1 mutant. Proc. Natl. Acad. Sci. U.S.A. 97, 8175-8179. doi 10.1073/pnas.100132197

Jouhet, J., Maréchal, E., Baldan, B., Bligny, R., Joyard, J., and Block, M. A. (2004). Phosphate deprivation induces transfer of DGDG galactolipid from chloroplast to mitochondria. J. Cell Biol. 167, 863-874. doi: 10.1083/jcb.200407022

Joyard, J., Maréchal, E., Miège, C., Block, M. A., Dorne, A. J., and Douce, R. (1998). "Structure, distribution and biosynthesis of glycerolipids from higher plant chloroplasts," in Lipid in Photosynthesis: Structure, Function and Genetics, eds P. A. Siegenthaler and N. Murata (Dordrecht: Kluwer Academic Publishers), 21-52. doi: 10.1007/0-306-48087-5_2

Kelly, A. A., and Dörmann, P. (2002). DGD2, an Arabidopsis gene encoding a UDP-galactose-dependent digalactosyldiacylglycerol synthase is expressed during growth under phosphate-limiting conditions. J. Biol. Chem. 277, 1166-1173. doi: 10.1074/jbc.M110066200

Kelly, A. A., Froehlich, J. E., and Dörmann, P. (2003). Disruption of the two digalactosyldiacylglycerol synthase genes DGD1 and DGD2 in Arabidopsis reveals the existence of an additional enzyme of galactolipid synthesis. Plant Cell 15, 2694-2706. doi: 10.1105/tpc.016675

Kobayashi, K., Awai, K., Nakamura, M., Nagatani, A., Masuda, T., and Ohta, H. (2009a). Type-B monogalactosyldiacylglycerol synthases are involved in phosphate starvation-induced lipid remodeling, and are crucial for lowphosphate adaptation. Plant J. 57, 322-331. doi: 10.1111/j.1365-313X.2008. 03692.x

Kobayashi, K., Nakamura, Y., and Ohta, H. (2009b). Type A and type B monogalactosyldiacylglycerol synthases are spatially and functionally separated in the plastids of higher plants. Plant Physiol. Biochem. 47, 518-525. doi: 10.1016/j.plaphy.2008.12.012

Kobayashi, K., Awai, K., Takamiya, K., and Ohta, H. (2004). Arabidopsis type B monogalactosyldiacylglycerol synthase genes are expressed during pollen tube growth and induced by phosphate starvation. Plant Physiol. 134, 640-648. doi: 10.1104/pp.103.032656

Kobayashi, K., Kondo, M., Fukuda, H., Nishimura, M., and Ohta, H. (2007). Galactolipid synthesis in chloroplast inner envelope is essential for proper thylakoid biogenesis, photosynthesis, and embryogenesis. Proc. Natl. Acad. Sci. U.S.A. 104, 17216-17221. doi: 10.1073/pnas.0704680104

Kobayashi, K., Masuda, T., Takamiya, K., and Ohta, H. (2006). Membrane lipid alteration during phosphate starvation is regulated by phosphate signaling and auxin/cytokinin cross-talk. Plant J. 47, 238-248. doi: 10.1111/j.1365313X.2006.02778.x

Kobayashi, K., Narise, T., Sonoike, K., Hashimoto, H., Sato, N., Kondo, M., et al. (2013). Role of galactolipid biosynthesis in coordinated development of photosynthetic complexes and thylakoid membranes during chloroplast biogenesis in Arabidopsis. Plant J. 73, 250-261. doi: 10.1111/tpj.12028

Lambers, H., Cawthray, G. R., Giavalisco, P., Kuo, J., Laliberté, E., Pearse, S. J., et al. (2012). Proteaceae from severely phosphorus-impoverished soils extensively replace phospholipids with galactolipids and sulfolipids during leaf development to achieve a high photosynthetic phosphorus-use-efficiency. New Phytol. 196, 1098-1108. doi: 10.1111/j.1469-8137.2012.04285.x

Lei, M., and Liu, D. (2011). Sucrose regulates plant responses to deficiencies in multiple nutrients. Plant Signal. Behav. 6, 1247-1249. doi: 10.4161/psb.6.8.16378

Li-Beisson, Y., Shorrosh, B., Beisson, F., Andersson, M. X., Arondel, V., Bates, P. D., et al. (2013). Acyl-lipid metabolism. Arabidopsis Book 11, e0161. doi: 10.1199/tab.0161

Maréchal, E., Block, M. A., Joyard, J., and Douce, R. (1994). Kinetic properties of monogalactosyldiacylglycerol synthase from spinach chloroplast envelope membranes. J. Biol. Chem. 269, 5788-5798.

Murphy, D. J. (1986). The molecular organisation of the photosynthetic membranes of higher plants. Biochim. Biophys. Acta 864, 33-94. doi: 10.1016/03044157(86)90015-8

Nakamura, Y., Arimitsu, H., Yamaryo, Y., Awai, K., Masuda, T., Shimada, H., et al. (2003). Digalactosyldiacylglycerol is a major glycolipid in floral organs of Petunia hybrida. Lipids 38, 1107-1112. doi: 10.1007/s11745-0061166-x

Nakamura, Y., Kobayashi, K., and Ohta, H. (2009a). Activation of galactolipid biosynthesis in development of pistils and pollen tubes. Plant Physiol. Biochem. 47, 535-539. doi: 10.1016/j.plaphy.2008.12.018

Nakamura, Y., Koizumi, R., Shui, G., Shimojima, M., Wenk, M. R., Ito, T., et al. (2009b). Arabidopsis lipins mediate eukaryotic pathway of lipid metabolism and cope critically with phosphate starvation. Proc. Natl. Acad. Sci. U.S.A. 106, 2097820983. doi: 10.1073/pnas.0907173106

Ohta, H., Shimojima, M., Arai, T., Masuda, T., Shioi, Y., and Takamiya, K. (1995). UDP-galactose: diacylglycerol galactosyltransferase in cucumber seedlings: purification of the enzyme and the activation by phosphatidic acid. Plant Lipid Metab. 152-155. doi: 10.1007/978-94-015-8394-7_42

Ohta, H., Yuzawa, Y., and Shimojima, M. (2012). Galactoglycerolipids. ASBMB Today 28-29. 
Okazaki, Y., Otsuki, H., Narisawa, T., Kobayashi, M., Sawai, S., Kamide, Y., et al. (2013). A new class of plant lipid is essential for protection against phosphorus depletion. Nat. Commun. 4, 1510. doi: 10.1038/ncomms 2512

Péret, B., Clément, M., Nussaume, L., and Desnos, T. (2011). Root developmental adaptation to phosphate starvation: better safe than sorry. Trends Plant Sci. 16, 442-450. doi: 10.1016/j.tplants.2011.05.006

Raghothama, K. G. (1999). Phosphate acquisition. Annu. Rev. Plant Physiol. Plant Mol. Biol. 50, 665-693. doi: 10.1146/annurev.arplant.50.1.665

Russo, M. A., Quartacci, M. F., Izzo, R., Belligno, A., and Navari-Izzo, F. (2007) Long- and short-term phosphate deprivation in bean roots: plasma membrane lipid alterations and transient stimulation of phospholipases. Phytochemistry 68, 1564-1571. doi: 10.1016/j.phytochem.2007.03.017

Sato, A., and Miura, K. (2011). Root architecture remodeling induced by phosphate starvation. Plant Signal. Behav. 6, 1122-1126. doi: 10.4161/psb.6.8.15752

Shimojima, M., Ohta, H., Iwamatsu, A., Masuda, T., Shioi, Y., and Takamiya, K. (1997). Cloning of the gene for monogalactosyldiacylglycerol synthase and its evolutionary origin. Proc. Natl. Acad. Sci. U.S.A. 94, 333-337. doi: 10.1073/pnas.94.1.333

Ticconi, C. A., and Abel, S. (2004). Short on phosphate: plant surveillance and countermeasures. Trends Plant Sci. 9, 548-555. doi: 10.1016/j.tplants.2004.09.003

Tjellström, H., Andersson, M. X., Larsson, K. E., and Sandelius, A. S. (2008). Membrane phospholipids as a phosphate reserve: the dynamic nature of phospholipid-to-digalactosyl diacylglycerol exchange in higher plants. Plant Cell Environ. 31, 1388-1398. doi: 10.1111/j.1365-3040.2008.01851.x

Versaw, W. K., and Harrison, M. J. (2002). A chloroplast phosphate transporter, PHT2;1, influences allocation of phosphate within the plant and phosphatestarvation responses. Plant Cell 14, 1751-1766. doi: 10.1105/tpc.002220

Wei, L., Miao, H., Zhao, R., Han, X., Zhang, T., and Zhang, H. (2013). Identification and testing of reference genes for Sesame gene expression analysis by quantitative real-time PCR. Planta 237, 873-889. doi: 10.1007/s00425-012-1805-9

Yamaryo, Y., Dubots, E., Albrieux, C., Baldan, B., and Block, M. A. (2008). Phosphate availability affects the tonoplast localization of PLDzeta2, an Arabidopsis thaliana phospholipase D. FEBS Lett. 582, 685-690. doi: 10.1016/j.febslet.2008.01.039
Yamaryo, Y., Kanai, D., Awai, K., Shimojima, M., Masuda, T., Shimada, H. et al. (2003). Light and cytokinin play a co-operative role in MGDG synthesis in greening cucumber cotyledons. Plant Cell Physiol. 44, 844-855. doi: $10.1093 / \mathrm{pcp} / \mathrm{pcg} 110$

Yamaryo, Y., Motohashi, K., Takamiya, K., Hisabori, T., and Ohta, H. (2006). In vitro reconstitution of monogalactosyldiacylglycerol (MGDG) synthase regulation by thioredoxin. FEBS Lett. 580, 4086-4090. doi: 10.1016/j.febslet.2006.06.050

Yuzawa, Y., Nishihara, H., Haraguchi, T., Masuda, S., Shimojima, M., Shimoyama, A., etal. (2012). Phylogeny of galactolipid synthase homologs together with their enzymatic analyses revealed a possible origin and divergence time for photosynthetic membrane biogenesis. DNA Res. 19, 91-102. doi: 10.1093/dnares/dsr044

Conflict of Interest Statement: The authors declare that the research was conducted in the absence of any commercial or financial relationships that could be construed as a potential conflict of interest.

Received: 30 July 2013; accepted: 30 October 2013; published online: 19 November 2013.

Citation: Shimojima $M$, Watanabe $T$, Madoka $Y$, Koizumi $R$, Yamamoto MP, Masuda K, Yamada K, Masuda $S$ and Ohta $H$ (2013) Differential regulation of two types of monogalactosyldiacylglycerol synthase in membrane lipid remodeling under phosphate-limited conditions in sesame plants. Front. Plant Sci. 4:469. doi: 10.3389/fpls.2013.00469

This article was submitted to Plant Nutrition, a section of the journal Frontiers in Plant Science.

Copyright (C) 2013 Shimojima, Watanabe, Madoka, Koizumi, Yamamoto, Masuda, Yamada, Masuda and Ohta. This is an open-access article distributed under the terms of the Creative Commons Attribution License (CC BY). The use, distribution or reproduction in other forums is permitted, provided the original author(s) or licensor are credited and that the original publication in this journal is cited, in accordance with accepted academic practice. No use, distribution or reproduction is permitted which does not comply with these terms. 